\title{
Deflectometric measurement of mechanical spectacle lens deformation
}

\author{
Assumpta Peral ${ }^{1}$, José Alonso ${ }^{1}$, Joán Canos Sanz ${ }^{2}$ and \\ Eusebio Bernabeu ${ }^{\dagger}$
}

${ }^{1}$ Departamento de Óptica, Universidad Complutense de Madrid. Avda. Arcos del Jalón s/n, 28037 Madrid, Spain, ${ }^{2}$ Departamento de Óptica, Universidad Complutense de Físicas, 28041 Madrid, Spain

\section{Summary}

The warpage of a set of six hard resin spherical lenses was measured when they were compressed by means of the tangential force exerted by a metal frame. The technique employed to measure the lenses improves over previous work both because greater precision is achieved, and because radii of curvature are measured in two perpendicular meridians. In this work, a representative sample of lenses has been used in order to test the way in which deformation depends on lens power and base. It is found that the induced warpage is enough to move the tested lenses between different base families, spoiling the initial design of the manufacturer. (c) 2000 The College of Optometrists. Published by Elsevier Science Ltd. All rights reserved.

\section{Introduction}

Plastic ophthalmic lenses can be deformed because of the compression exerted by the frame, the effect being even more noticeable when the lenses are exposed to a heat source. Characterisation of this deformation, and its quantitative relation to the force exerted by the rim, is important in order to predict whether a determined level of applied force is acceptable from the optical point of view. Other factors may produce lens deformation, for example CR-39 shrinks as it ages, and the consequent reduction in volume produces an increase in the radius of curvature of the surfaces of a lens made with this material, as reported by Kislin et al. (1972). Smith and Wientzen (1978) studied the visual effects caused by a cylindrical warpage of $1 \mathrm{D}$ of the spectacle lens surface, which is the maximum tolerance specified in the prescription lens standard ANSI Z 80-1-72. Dowaliby et al. (1980) used a lens gauge to measure the bitoricity of hard resin lenses induced by the pressure exerted by the frame. They measured the lenses of a sample of 275

\footnotetext{
Received: 2 August 1999

Revised form: 7 November 1999

Accepted: 20 November 1999
}

Correspondence and reprint requests to: A. Peral. Tel.: +34-1-394-4555; fax: $+34-1-394-4678$. completed spectacles chosen at random. Metal frames produced deformation exceeding the ANSI standard in 7\% of the lenses. Other frame materials yielded smaller deformations. In a previous work (Alonso et al., 1997), we proposed a linear model that, for spherical lenses, relates the radius of curvature to the force exerted by the rim. This model allows us to determine the force required to induce a fixed level of warpage, at least in a circular frame. Also, some general conclusions were reached about the deformation of a lens according to its power. For example, at the same level of applied force, deformation in the paraxial region is more important in negative lenses than in positive ones. This behaviour is to be expected, since the centre thickness is greater in positive lenses. Nevertheless, our previous results indicate that not only the thickness, but also the base curve, of the lens affects the degree of induced deformation. One of the objectives of the present work was to test this hypothesis. For this reason we used six CR-39 spherical lenses from the same manufacturer, with refractive index $n=1.523$ and powers evenly spaced from -2.5 to $-6 \mathrm{D}$ and distributed in two different base values, +2.20 D and $+2.96 \mathrm{D}$ (we define base in this work as the refracting power of the front surface of the lens). We have measured the centre thickness, found to be $e=1.25 \pm 0.02 \mathrm{~mm}$ for all the lenses.

In this study, we employed a deflectometric technique 


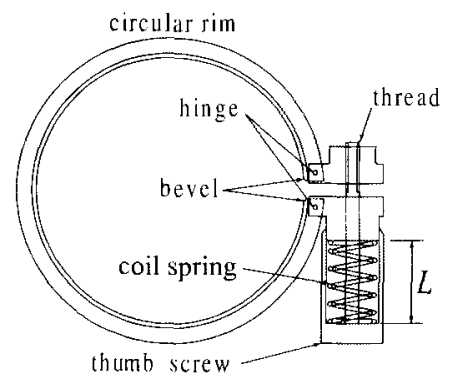

Figure 1. Frame with the pressing mechanism that we have employed to apply a controlled amount of tangential force to the rim. The force is converted into a radial strain field in the lens.

that allowed us to obtain higher accuracy and resolution in the measurement of radius of curvature. The technique is a modification of an autocollimation method in which beam divergence is measured by means of the moire fringe pattern generated by two crossed Ronchi gratings. The precision achieved depended on the value of the measured radius, but in all of our samples, it lies in the interval 0.05$0.3 \mathrm{~mm}$, which was about ten times better than that achieved in our previous work. Another advantage of the technique is that it permits us to measure of the radius of curvature along two different meridians. Although the measured lenses were spherical, we found a lack of revolution symmetry on their surfaces, probably due to manufacturing errors. With this deflectometric method we have been able to measure these errors and how they change as compression is applied to the lenses. Other deflectometric techniques for testing spectacle lens and mould surfaces are nowadays routinely used in some manufacturing companies, with similar accuracy to and better measuring time than those obtained with classical profilometry.

\section{Method}

Compression was applied to the set of spherical lenses by means of the circular aluminium frame shown in Figure 1. The inner diameter of the frame is $55 \mathrm{~mm}$. The centre of the lenses is located with an automatic focimeter. After bevelling, we have measured the distance from the optical centre to the geometrical centre. In all the lenses the decentration was found to be less than $0.5 \mathrm{~mm}$. As the thumbscrew of the pressing mechanism of the frame is rotated, the coil spring is compressed. The spring applies a tangential force to the rim that is converted to a radial strain field by means of the hinges that connect the rim and the pressing mechanism. The inner edges of the rim are bevelled at the points indicated in Figure 1 with the label bevel, in order to avoid these corners of the rim distorting the revolution symmetry of the compressed lens. That way we ensure adequate boundary conditions. Spring elongation is measured by means of a nonius scale on the thumbscrew, and the maximum tangen-

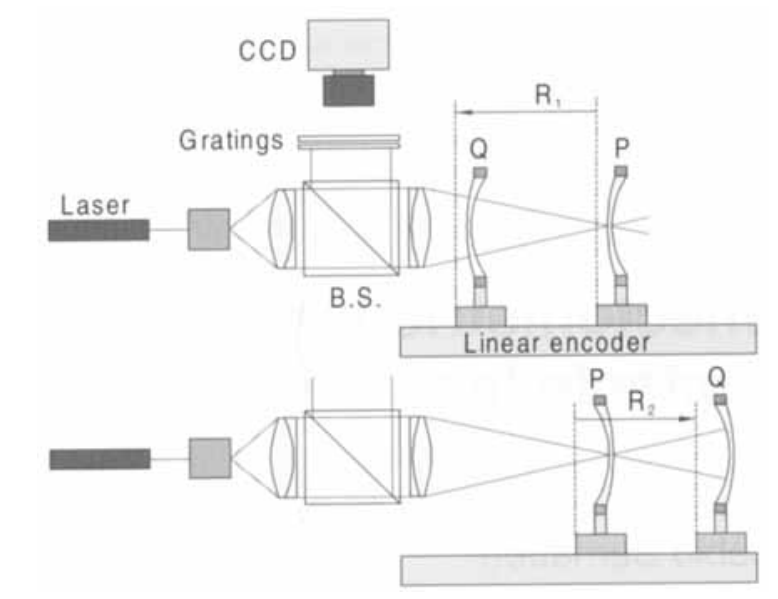

Figure 2. Experimental set-up employed for the measurement of mechanical warpage in two perpendicular meridians.

tial force we can apply is about $150 \mathrm{~N}$. As previously demonstrated, we can achieve similar force levels in sturdy metal frames, but not in plastic frames. The experimental setup intended for measuring the paraxial radius of curvature of both lens surfaces is shown in Figure 2. The measuring principle is similar to that of a radioscope for contact lenses, but instead of looking for the best image formation of a reticule, we look for the best beam collimation. A laser beam is spatially filtered and collimated by a high quality doublet. Next, a converging beam is produced with another high quality doublet with a focal length of $300 \mathrm{~mm}$. At the position denoted by $P$ in Figure 2, the beam is brought to a focus on the surface vertex. At position $Q$, the radius of curvature of the beam coincides with the radius of curvature of the lens surface. Thus the radius of curvature of the surface equals the displacement of the lens between the positions $P$ and $Q$, for which collimation of the reflected beam is restored after passing back through the second doublet. The displacement is measured with an accuracy of $\pm 1 \mu \mathrm{m}$ by means of a glass scale linear encoder attached to the optical bench. To test collimation, we use moiré deflectometry (Kafri and Glatt, 1990). A beam splitter deviates the beam and directs it upon two gratings which form a moiré fringe pattern. The resulting image is captured and analysed by means of a CCD camera connected to a frame grabber. The period of the two gratings is $50 \mu \mathrm{m}$, and the second one is located at the third Talbot plane of the first one (that is, $11.852 \mathrm{~mm}$ for the wavelength of the $\mathrm{He}-\mathrm{Ne}$ laser) in order to achieve maximum sensitivity to the convergence or divergence of the returning beam (Keren and Kafri, 1985).

Initially, an optical quality plane mirror replaces the second doublet, and the position of the first doublet is varied until the beam that is incident upon the gratings is perfectly collimated. The angle between the gratings is adjusted in such a way that six to ten fringes are observed within the field of view. We then replace the mirror with the second 


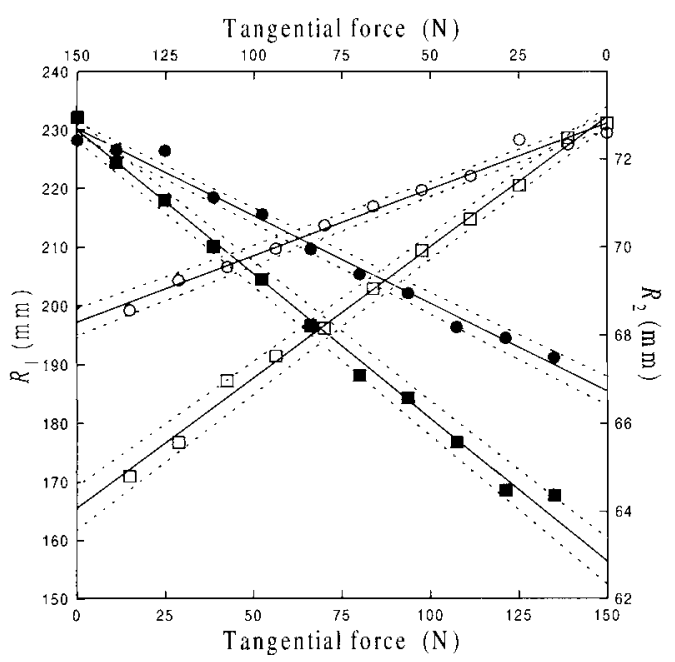

Figure 3. Relationship between radii of curvature and tangential force. Hollow symbols correspond to the radii of curvature of the front surface, whereas solid symbols correspond to those on the back surface. Circles correspond to the radii of curvature of the flatter meridians. Squares correspond to the radii of the stepper meridians. Solid lines show the linear fit to the data. Error corridors are shown with dashed lines. The horizontal scale for $R_{2}(T)$ (upper horizontal axis) is inverted in order to avoid graph overlapping.

doublet and locate the spectacle lens so that the beam focus is near its front surface. Unless the beam is exactly focused on the front surface, the reflected beam that is incident on the gratings is not perfectly collimated so we observe a change of the period of the moire fringes as well as a change in their orientation. When the lens is located exactly at points $P$ and $Q$, collimation of the reflected beam is restored and the fringes recover their original period and orientation. Because of the high resolution and accuracy of the linear encoder, the overall resolution of the method is limited by the ability of the observer to restore the original fringe pattern. The error is basically of random nature and we have determined each radius by averaging 15 measurements of the distance between $P$ and $Q$. The standard deviation obtained, and henceforth the mean quadratic error, $\Delta R$, depends on the radius of curvature, but we have obtained values of $\Delta R$ in the interval $0.06-0.8 \mathrm{~mm}$, as stated in the introduction. In this way, we have improved the accuracy of our previous measurements of radius of curvature (Alonso et al., 1997) by one order of magnitude.

At position $Q$ the beam reflects on a large portion of the lens surface, so variations in the shape of the surface from the spherical form can be observed as deformations of the fringe pattern. Usually, the recovery of the initial period and fringe orientation can only be obtained at the central region of the field of view (paraxial region). Deformations of the fringe pattern outside this area are related to aberrations of the reflected beam, which in turn are related to surface deformation.
The deflectometer only tests for collimation in the direction perpendicular to the moire fringes. We have therefore made measurements of the radius of curvature in the two orthogonal meridians of maximum and minimum curvature. In order to do this, the lens was rotated around its optical axis, and the largest and smallest radius of curvature were determined. As we will present in the next section, some lenses showed some cylinder on both surfaces, but this cylinder appeared or changed in all the lenses as they were compressed.

\section{Results}

As mentioned above, the relationship between the radius of curvature of centred surfaces of revolution and the tangential force applied to the frame is linear within a good approximation. As long as we suppose that the spherical shape of the lens surfaces is preserved under any realistic applied tangential force, we just need one parameter, $\xi$, to describe the effect of this tangential force on the surface geometry,

$R_{i}(T)=\xi_{i} T+R_{i}(0)$.

$R_{i}(0)$ is the initial radius of curvature, $R_{i}(f)$ is the radius in the presence of the applied tangential force of magnitude $T$, and $\xi_{i}$ is a constant depending on the lens power and form. A linear fit to the experimental data and error corridors are presented in Figure 3 for the lens 6. Maximum and minimum values of the radius of curvature of the front surface vs. tangential force are plotted using the vertical axis at the left and the lower horizontal axis. The radii of curvature of the back surface vs. tangential force are plotted using the vertical axis at the right and the upper horizontal axis, which is inverted to avoid overlapping of different traces. We assume that the measured values of the radius of curvature are normally distributed. Under this assumption, standard statistical tools allow us to compute the errors in the fitting parameters, slope and intersection point with the $y$-axis. Using the error of the fitting parameters, we can compute the confidence intervals for the predicted radius of curvature, and the extreme of these intervals is aligned on the lines that delimit the error corridor.

The parameters of the linear model, $R_{i}(0)$ and $\xi_{i}$, that we have obtained for the six lenses employed are presented in Table l. We have computed the back vertex power along the principal meridians by means of the radii of curvature $R(0)$ of the undistorted lenses, along with the refractive index and centre thickness. The averaged back vertex power is shown in the first row. The results obtained are in good agreement with the values of the back vertex power measured with an automatic focimeter, which are presented in the second row of Table 1. A graphical representation of the measured parameters is shown in Figure 4. The radius of curvature of each surface without any applied tangential force is shown in Figure $4(a)$. The white and black bars correspond to two 


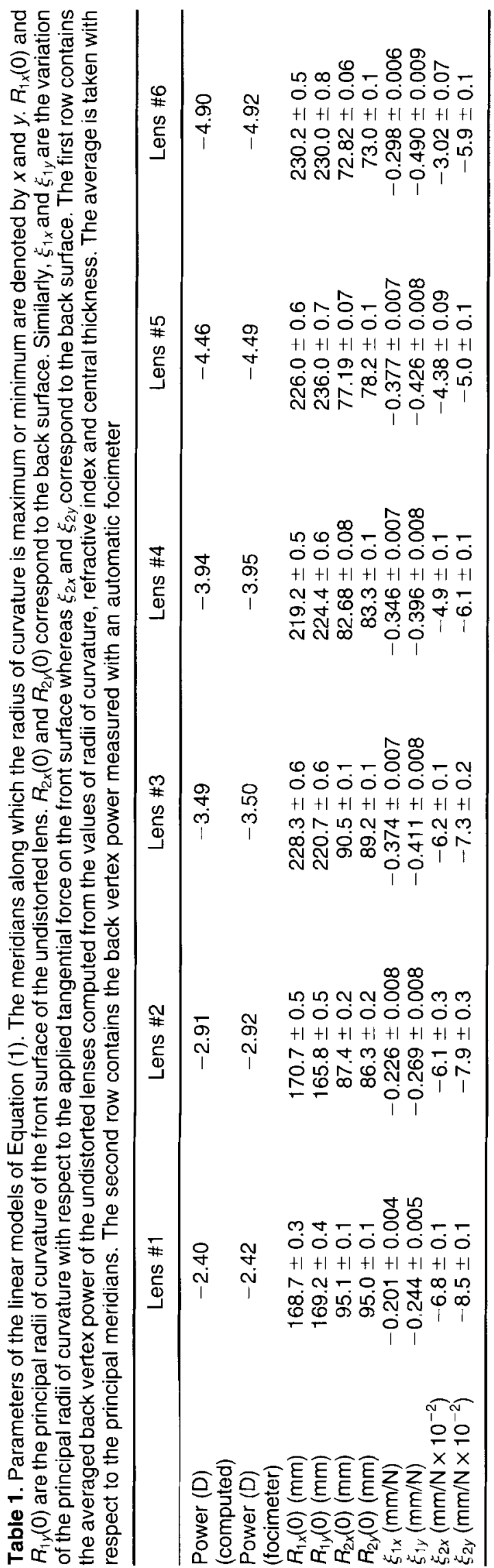

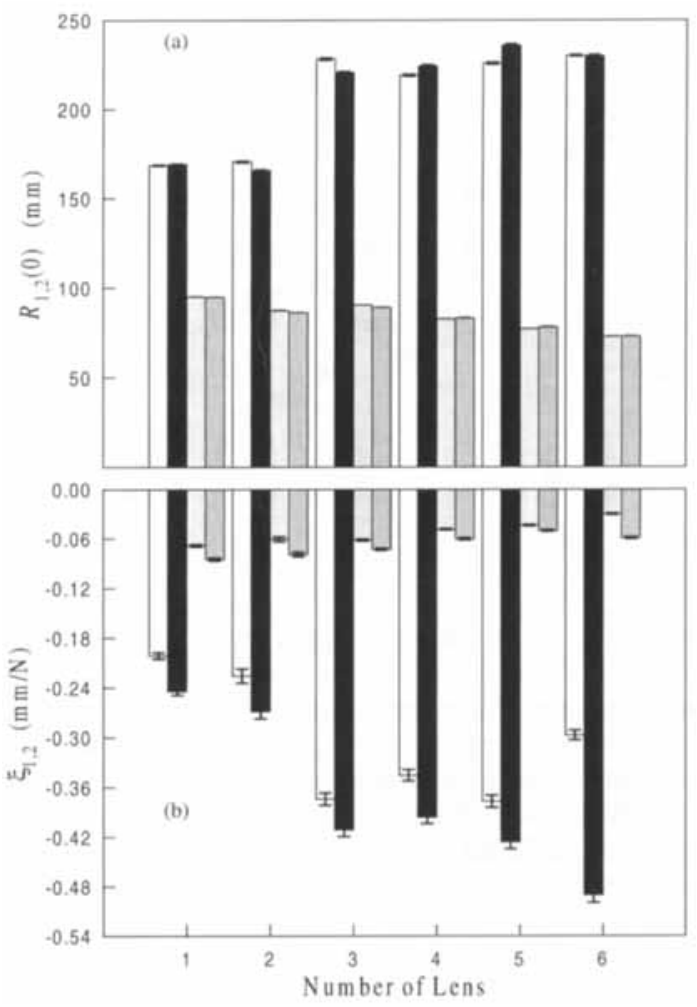

Figure 4. Parameters of the linear model of Equation (1). (a) Radius of curvature of both surfaces in two orthogonal meridians for the undistorted state. (b) Coefficients of variation of the radius of curvature corresponding to each orthogonal meridian and each surface. In both graphs, white and black bars correspond to the parameters of the front surface. Grey bars correspond to the parameters of the back surface.

orthogonal meridians of the front surface (base) and the light and dense grey bars to the corresponding meridians on the back surface. It can be observed that the lenses 1 and 2 are made on base $2.96 \mathrm{D}$, and lenses 3-6 are made on base $2.20 \mathrm{D}$. Also, we can see that although the lenses are spherical, because of manufacturing errors they exhibit some cylinder on both surfaces even without any applied tangential force. This cylinder can be noticed particularly at the front surface. The difference between the orthogonal curvature radiuses is higher in lenses 3 and 5 , the last one being about $10 \mathrm{~mm}$. As we can see in lenses 1 and 6 , this variation is quite small at the back surface. The observed surface astigmatism does not significantly affect the vertex power of the lenses because the cylinder on both surfaces is almost the same, with similar orientation but with opposite signs.

Figure $4(b)$ shows the coefficients of radius of curvature variation $\xi_{i}$ expressed in $\mathrm{mm} / \mathrm{N}$. The white and black bars refer to the same two orthogonal meridians on the front surface whose radii are presented in Figure $4(a)$. Grey (light and dark) bars refer to the coefficients for the back surface. In our previous study on lens deformation it was 
suggested that the larger the radius of curvature is, the larger its variation for any level of applied force. In the present work we confirm this statement. For any of the six lenses, the mean coefficient of radius of curvature variation satisfies $\left|\xi_{1}\right|<\left|\xi_{2}\right|$. With respect to this parameter, the distribution of lenses on two different base values can be observed. Lenses 1 and 2 present a mean coefficient of variation $\xi_{1}=-0.235 \mathrm{~mm} / \mathrm{N}$ and the same parameter for lenses 36 is $\xi_{2}=-0.389 \mathrm{~mm} / \mathrm{N}$.

The coefficient of variation $\xi_{i}$ also depends on the orthogonal meridians of maximum and minimum power. For example, the variation of these coefficients between the orthogonal meridians of the front surface of lenses $1-5$ is in the range $0.037-0.050 \mathrm{~mm} / \mathrm{N}$, which is a relative variation of $18 \%$ for lenses 1 and 2 (base $2.96 \mathrm{D}$ ) and $12 \%$ for lenses $3-5$ (base $2.20 \mathrm{D}$ ). Lens 6 behaves differently from this rule because we observe a variation of $\xi_{1}$ of $0.192 \mathrm{~mm} /$ $\mathrm{N}$, which is $40 \%$ of the greatest value. A similar behaviour is observed in the back surface of this lens, as is shown in Figure $4(b)$. This effect could be due to a lack of homogeneity in the bulk material of the lens or a defect in the edging process that could produce a slightly elliptical shape instead of a perfect circular one. In this last case, the circular frame would apply the main compression along the meridian corresponding to the long axis of the elliptical contour. This effect could explain the large difference between the coefficients of radius of curvature variation.

Next, we analyse the effect that the deformation could have on the optical performance of the lens. Since the lens bending has increased, a variation in the correction of oblique astigmatism and power error is expected. Also, variation of both dynamic and static distortion will occur. As a general rule, modern ophthalmic lenses are shallower than the full correction of oblique astigmatism would require. Also, minimum distortion (both dynamic and static) requires far steeper bases than those usually employed. This is because lens weight and aesthetic factors are taken into account in the physiological merit functions that nowadays are employed in the design of monofocal ophthalmic lenses.

We have analysed the off-axis performance of the measured lenses. For this, we have averaged the refractive powers in each of the orthogonal meridians of maximum and minimum curvature, and we have carried out the calculations assuming the surfaces are spherical with the radius of curvature that results from the averaging process. The results for all the tangential force levels applied are presented in Figure 5. At any of these levels, lenses are represented by points in the plane $F, F_{1}$, where $F$ is the back vertex power and $F_{1}$ is the refractive power of the front surface. Each lens is easily identified by its back vertex power in the horizontal coordinate. The point at the bottom of each set represents the shallower lens form, that is, the form factor that we have measured without any applied tangential force. As tangential force augments, the point representing each lens moves towards higher values of $F_{1}$, which is a graphical interpretation of the bending increase. As pointed out in our first study (Alonso et al., 1997), back vertex power is almost constant as compression increases, because the refractive power increase in the two lens surfaces is almost the same. This can be observed in Figure 5, in which all the points corresponding to each lens are lined up vertically.

By means of exact ray tracing (Jalie, 1992), oblique astigmatism (Figure 5(a)) and power error (Figure 5(b)) have been computed by us for the following standard parameters: obliquity angle $u_{2}^{\prime}=30^{\circ}$, distance from the back surface to the centre of rotation of the eye $l_{2}^{\prime}=27 \mathrm{~mm}$ and object vergence $L=0$. The value of the off-axis aberration is encoded with a greyscale colour map, and some contour lines are added to improve quantitative comparison. It can be seen in both Figure 5(a) and (b) that all the lenses exhibit, in their original state, some oblique astigmatism and power error. The absolute value of oblique astigmatism is about $0.2 \mathrm{D}$ in lenses 1 and 2 , and about $0.3 \mathrm{D}$ in the remaining lenses. The power error for all the lenses is in the range -0.05 to $-0.15 \mathrm{D}$. As tangential force increases, the oblique astigmatism decreases in all the lenses, getting close to $-0.1 \mathrm{D}$ in lenses 1 and 2 and around $-0.15 \mathrm{D}$ in the remaining lenses. With respect to power error, lenses 2 and 5 change to the Percival form at the larger value of tangential force, whereas lens 6 changes to the Percival form at the eighth value of tangential force. In all the cases, distortion will decrease as the lens bending increases. Nevertheless, the effect will be quite small, because the bases for which both dynamic and static distortion are minimum are above $20 \mathrm{D}$ for the powers and refractive index involved.

With respect to the aberrations analysed herein, and assuming that the spherical shape is preserved, the potential user would be expected to observe a small increase of acuity when sighting off-axis. If the user could compare two similar lenses with the same manufacturing base, one of them being compressed, some differences in the perception could be noticed, because of the small changes in distortion, retinal image size and visual acuity. Anyway, the user would be expected to adapt to the new conditions within a few days, as normally occurs when replacement lenses with different bases are prescribed and mounted.

Other aberrations that could appear are associated with the departure of the surface shape from the spherical form. In effect, we have seen that a surface cylinder may appear or change when compression is applied. For example, in lens4 we measured an initial cylinder of almost $0.1 \mathrm{D}$ in each surface. After applying compression, it grew to $0.6 \mathrm{D}$. In lens 6 we got similar values of surface cylinder after compression, but the initial values were smaller than the experimental error, which is $\pm 0.03 \mathrm{D}$. The measured cylinders at each surface almost cancel each other out, so the on-axis astigmatism is small $(-0.17 \mathrm{D}$ in lens 6 at the maximum tangential force level), but the off-axis 

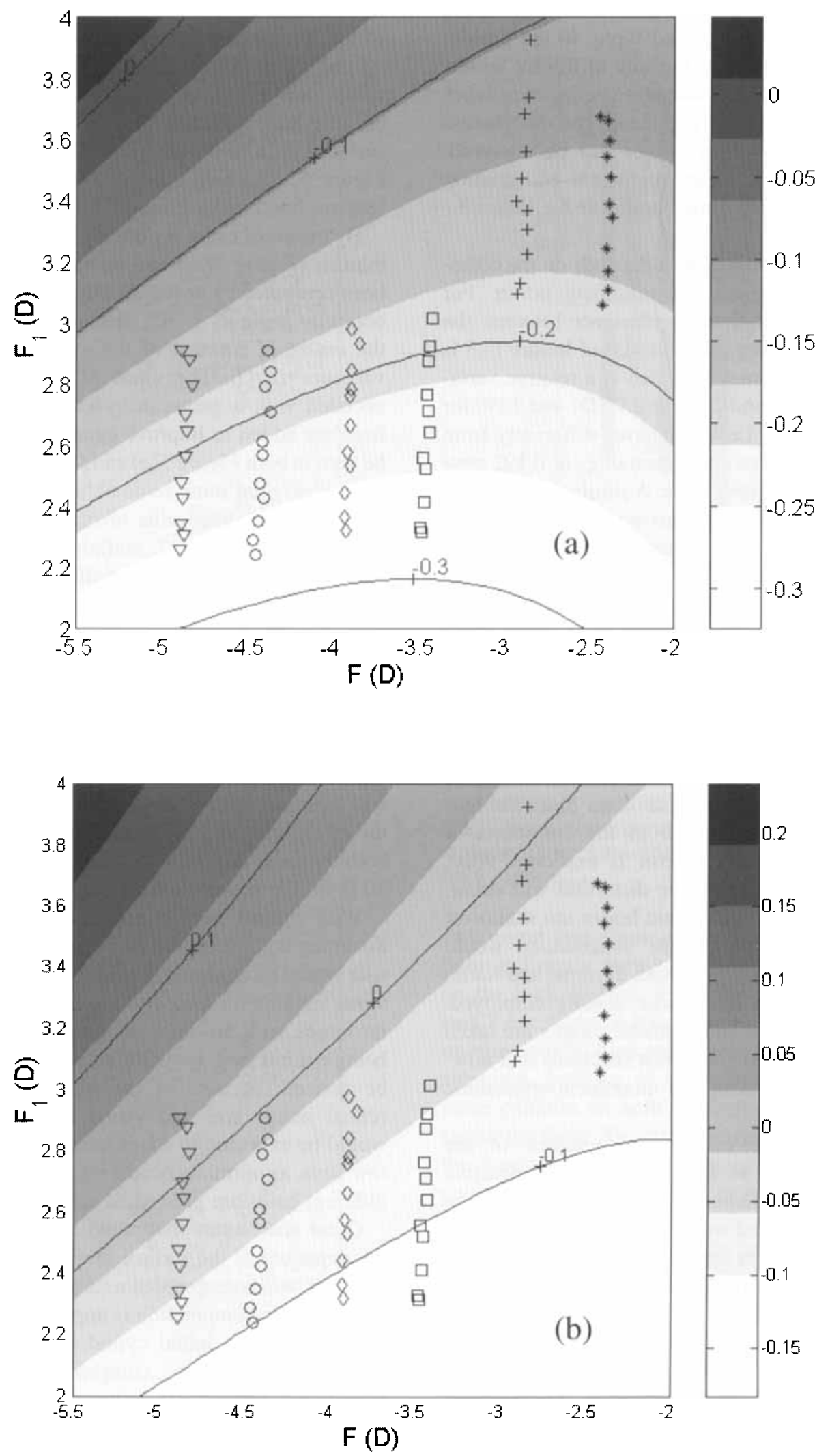

2

15
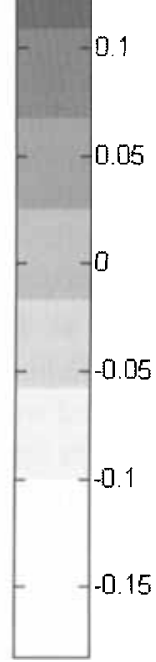

Figure 5. Off-axis performance at $30^{\circ}$ of the analysed lenses. (a) Oblique astigmatism. (b) Power error. In each case, the lowest point represents the result with the undistorted lens. 
astigmatism will be modified with respect to the values obtained by considering spherical surfaces. Visual inspection of the moiré fringes indicates that strong aspherization does occur at the outer regions of the lens surfaces, which confirms our previous results. Other types of non-uniform deformations can be expected, especially when the rim of the frame is non-circular and the compression is exerted in lens regions with different thickness. Unfortunately, the method that we have used only allows us to obtain the central radius of curvature of each surface. We do not have enough information about local surface deformation to proceed to a detailed ray tracing calculation that could provide us with quantitative information.

\section{Discussion}

We have improved some aspects of previous measurements on ophthalmic lens deformation due to mechanical compression. We have used a more precise, moiré-based technique to measure a set of lenses with back vertex powers evenly spaced from -2.5 to $-6 \mathrm{D}$. Negative lenses were chosen because they are more susceptible to deformation than positive lenses, as demonstrated in our first study on this subject. According to the base, the lenses are grouped in two different sets, so the effect of the base can be taken into account when compression changes the form factor of the lenses. We have confirmed that the flatter the surface, the higher the deformation. If we consider lenses with different powers but with the same base, the greater the power, the smaller the radius of curvature variation on the back surface.

We have observed that the maximum compression exerted on the lenses (which is similar to the compression achievable with a sturdy metal spectacle frame) can move the lenses between different base sets. This effect could spoil the manufacturer's design. In particular, the practitioner should avoid excessive compression in the plastic lens mounting process to ensure quick and comfortable adaptation by the wearer to the prescription.

Finally, the surfaces of the lens under compression will be forced out of the spherical shape. In all the measured lenses, surface cylinder changes when tangential force is applied. In some samples the cylinder grows with the compression, while in the other samples the surface cylinder decreases as tangential force increases. The surface cylinder produces bitoricity, although in our lenses this did not create a significant value of astigmatism on the lens axis. Nevertheless, the bitoricity will undoubtedly affect the off-axis performance of the lenses. Strong aspherization has also been observed in the fringe patterns resulting from the moiré technique that we have employed.

\section{Acknowledgements}

We thank T. Yonte for her teaching in the use of the moiré deflectometer. We also thank $\mathrm{C}$. Illueca for his suggestions. This work has been supported by the project TAP980701/97, from the Comisión Interministerial de Ciencia y. Tecnologia of Spain.

\section{References}

Alonso, J., Peral, A., Sanz, J. C. and Bernabeu, E. (1997). Measurement of mechanical warpage in CR-39 lenses. Ophthal. Physiol. Opt. 17, 81-87.

Dowaliby, M., Nichols, E. and Bailey, M. E. (1980). Comparative study involving bitoric effect of hard resin lenses mounted in optyl frames, zylonite frames, and metal frames. Am. J. Optom. Physiol.Opt. 57 (2), 109-112.

Jalie, M. (1992). The Principles of Opthalmic Lenses, 4 th ed., The Association of British Dispensing Opticians, London.

Kafri, O. and Glatt, I. (1990). The Physics of Moiré Metrology, Wiley, New York.

Keren, E. and Kafri, O. (1985). Diffraction effects in moiré deflectometry. J. Opt. Soc. Am. A 2, 111-120.

Kislin, B. et al. (1972). Warpage of CR-39 Plastic Lenses. Brooks Air Force Base, Texas, USAF School of Aerospace Medicine, SAM-TR-72-4, July 1972.

Smith, F. D. and Wientzen, R. V. (1978). Prediction of visual effects from the warpage of spectacle lenses. J. Optom. Arch. Am. Acad. Optom. 616-631. 